\title{
Diversity by Design
}

$\mathrm{T}$ The September 2013 issue of the Journal of Graduate Medical Education includes a study by Auseon and colleagues, " "Successful Efforts to Increase Diversity in a Cardiology Fellowship Training Program" that profiles an initiative to attract more underrepresented minority physicians into a university-based cardiovascular medicine fellowship. The piece contributes to a sparse but growing body of literature regarding promising practices for creating diverse and inclusive residency training programs. ${ }^{2}$

Much of the discourse regarding diversity in medicine has focused on ways to increase the pipeline of minority students prepared for and accepted into undergraduate medical education and, to a lesser extent, increasing the number of minority faculty members at the nation's medical schools. These are both critical targets, although efforts to diversify the physician workforce will not be complete without a deliberate focus on graduate medical education. Residency is a significant phase in the development of every physician, where practices become ingrained and the values of humanism, cultural competence, and empathy are sensitive to reinforcement or erosion. ${ }^{3}$ Institutional diversity can play a supportive role in these processes by exposing residents to the value of different perspectives, providing opportunities to hone cross-cultural awareness, and enhancing the overall learning environment. Little research has been published that examines effective interventions, making this new study both timely and important.

Medical schools and teaching hospitals have developed initiatives aimed at increasing the number of minorities and other marginalized groups in medicine for more than 40 years, and yet we still have not achieved a high level of precision in differentiating an effective intervention from a marginal or ineffective one. With the country's demographics undergoing a dramatic shift, accompanied by the impending expansion in access to care through health care reform, the time has come to amplify and accelerate our efforts to create a physician workforce that is more reflective of and responsive to the needs of all patients.

Both authors are with the Association of American Medical Colleges. Marc A. Nivet, EdD, is Chief Diversity Officer and Anne C. Berlin, MA, is Senior Specialist for Outreach.

Corresponding author: Marc A. Nivet, EdD, Association of American Medical Colleges, 2450 N Street NW, Washington, DC 20037, 202.741.6478,

ChiefDiversity@aamc.org

DOI: http://dx.doi.org/10.4300/JGME-D-13-00200.1
Auseon and colleagues ${ }^{1}$ add valuable evidence to the conversation on what works, which begins with engaged leadership at the highest level committing both human and financial resources to diversity as a strategic priority. The profiled initiative establishes that supportive networks and affinity groups to combat the social isolation of minorities and other marginalized groups-a tested approach for medical students and faculty-are also effective in residency settings. ${ }^{4}$ Further, this study demonstrates the value that prospective residents and fellows place on tailored professional development and mentorship opportunities as a sign that an institution is supportive and welcoming. Perhaps the most valuable contribution is the sheer fact that in shaping their diversity initiative, the authors recognized the importance of moving away from a simple passive reception of those diverse applicants who apply to an active engagement in ensuring that diverse candidates apply and are made aware of the institution's strategic commitment to diversity. The diversity we seek must happen by design.

Further studies should build on this important work by considering the following:

\section{Connect resident diversity with medical student and} faculty initiatives Student, resident, and faculty diversity go hand in hand. Underrepresented minority undergraduate medical students look to underrepresented minority faculty as role models in their career development, and residents evaluate faculty diversity and satisfaction as a signal of institutional culture, for instance. ${ }^{5}$ Because of these interrelations, economies of scale are available to institutions that link existing diversity-related pipeline and faculty development programs institution-wide. This case study highlights the importance of encouraging diversity earlier in the pipeline; at present, residency and fellowship programs are fighting over a finite pool of diverse physicians. As the authors point out, we need more underrepresented minority cardiologists to tackle the intractable health disparities related to cardiovascular disease. At the same time, we also need more underrepresented minority primary care physicians; thus, we need to broaden the full pool.

Consider your institutional culture We know residency is a time of great challenge for all people given the rigors and pace of work, but being viewed as an outlier or special recruit may subject minority residents to undue pressure and additional stressors. Investments in recruitment should be enhanced and reinforced by efforts to promote an overall culture and climate of inclusion where all can 
thrive. A positive institutional culture will serve as a beacon within the informal communication channels that prospective residents and fellows use to determine their choice of program.

Diversity efforts should be data-driven Those of us who are steeped in diversity efforts recognize that we are in a pinch: these intentional recruitment efforts are needed more than ever to meet the needs of the diverse patients of the 21st century, yet such efforts are increasingly constrained given the financial challenges we are all facing. The time has come for us to determine what elements of our existing institutional diversity strategies are effective and which are not, and to have the courage to strategically abandon efforts that are marginal at best while concentrating resources on the most promising interventions. ${ }^{6}$ To do this, additional studies are required to triangulate and ensure that the success of this university-based program is repeatable and transferable.
Finally, further research should incorporate metrics for success beyond compositional diversity over time, such as linking workforce diversity efforts back to quality improvements and minimizing or eradicating health inequities.

\section{References}

1 Auseon AJ, Kolibash AJ Jr, Capers Q IV. Successful efforts to increase diversity in a cardiology fellowship training program. J Grad Med Educ. 2013;5(3): $481-485$

2 Liebschutz JM, Darko GO, Finley EP, Cawse JM, Bharel M, Orlander JD. In the minority: black physicians in residency and their experiences. J Natl Med Assoc. 2006;98(9):1441-1448.

3 Markakis KM, Beckman HB, Suchman AL, Frankel RM. The path to professionalism: cultivating humanistic values and attitudes in residency training. Acad Med. 2000;75(2):141-150.

4 Dickins K, Levinson D, Smith SG, Humphrey HJ. The minority student voice at one medical school: lessons for all? Acad Med. 2013;88(1):73-79.

5 Page KR, Castillo-Page L, Wright SM. Faculty diversity programs in U.S. medical schools and characteristics associated with higher faculty diversity. Acad Med. 2011;86(10):1221-1228.

6 Nivet, MA. Commentary: diversity and inclusion in the 21st century: bridging the moral and excellence imperatives. Acad Med. 2012;87(11):1458-1460. 